$($ LGAIN $=14.1 ; p=0.017)$. This difference was primarily driven by an increase in Treg frequencies (HGAIN $=4.00 \%$, $\mathrm{LGAIN}=2.38 \%$, No $\mathrm{AIN}=1.88 \% ; \mathrm{p}=0.048)$, and participants with HPV16/18 also had significantly higher Treg frequencies (HPV16/18 = 4.02\%, No HPV16/18 = 2.68\%; $\mathrm{p}=$ $0.021)$. Th17 frequencies did not vary with HPV status, HPV16/18 positivity or AIN stage. No mucosal parameters were associated with anorectal HIV RNA detection.

Conclusion HPV infection and high-grade AIN were associated with diffuse mucosal Treg infiltration, reducing the mucosal Th17/Treg ratio in participants with high-grade AIN. While changes in $\mathrm{T}$ cell immunology did not increase HIV RNA shedding in ART+ MSM, their effect on ART-naïve men or HIV susceptibility in HIV-negative men will be interesting areas for future research.

Disclosure No significant relationships.

\section{P156 STUDY ON THE EFFECT OF SLANDC-MEDIATED ADCC IN HIV-1-INFECTED PATIENTS}

${ }^{1}$ Qian Jiang, ${ }^{2} \mathrm{H}$ Sun, ${ }^{1}$ Hualu Cui, ${ }^{1}$ Wenqing Geng*, ${ }^{1}$ Hong Shang. ${ }^{1}$ The First Affiliated Hospital, China Medical University, Shenyang, China; ${ }^{2}$ AIDS Institute, China Medical University, Shenyang, China

10.1136/sextrans-2019-sti.319

Background Antibody-dependent cell-mediated cytotoxicity (ADCC) mediated by natural killer (NK) cells plays a critical role in HIV-1 infection. As a novel subset of dendritic cells (DCs), 6-sulfo LacNAc-expressing DCs (slanDCs) also express CD16. However, the levels of slanDC-mediated ADCC during HIV-1 infection are not well addressed.

Methods Forty-five HIV-1-infected subjects were enrolled and 19 HIV-1 negative individuals were used as healthy controls (HCs). The complex of gp120 and anti-gp120 was used to stimulate peripheral blood mononuclear cells (PBMCs) and the level of TNF-alpha secreted by slanDCs was detected using intercellular staining of flow cytometer.

Results The counts of slanDCs in HIV-1-infected and treatment naïve patients were significantly lower than those of $\mathrm{HCs}$ and those receiving anti-retrovirus therapy (ART) $(\mathrm{P}=0.0331, \mathrm{P}<0.0001)$. The number of slanDCs in HIV-1infected patients with ART was significantly higher than those who did not receive ART, indicating that ART could help HIV-1-infected individuals to recover the number of slanDCs. The level of slanDC-medated ADCC evaluated as the level of TNF-alpha production by slanDCs stimulated by the complex of gp120-anti-gp120, was significantly lower in HIV-1-infected subjects as compared with $\mathrm{HCs}$ and those receiving ART $(\mathrm{P}=0.0011, \mathrm{P}=0.0002)$. The expression of CD16 (MFI) by slanDCs from HIV-1-infected patients receiving ART was significantly higher than that from HIV-1-infected untreated and HCs $(\mathrm{P}=0.0014, \mathrm{P}=0.0003)$, and the expression of CD16 (MFI) in slanDC was positively correlated with the ADCC effect $(\mathrm{P}<0.0001)$.
Conclusion The slanDC-medated ADCC existed in HIV-1infected patients and the level could be enhanced by ART, suggesting an alternative pathway involved in ADCC in HIV-1 infection.

Disclosure No significant relationships.

\section{P157 CROWDSOURCING METHODS TO ENHANCE HIV AND SEXUAL HEALTH SERVICES: A QUALITATIVE SCOPING REVIEW OF EVIDENCE}

${ }^{1}$ Dan Wu, ${ }^{2} J a s o n$ Ong, ${ }^{3}$ Tiarney Ritchwood, 'Weiming Tang*, ${ }^{1}$ Joseph Tucker. ${ }^{1}$ UNC Project-China, Guangzhou, China; ${ }^{2}$ LSTHM, London, UK; ${ }^{3}$ Duke University, Durham, USA

\subsection{6/sextrans-2019-sti.320}

Background Crowdsourcing, a process where non-experts and experts work together to solve a problem, may be useful for developing HIV and sexual health interventions, programs, and research. This qualitative scoping review examines evidence on crowdsourcing to improve HIV and sexual health services.

Methods We searched four databases in June 2018. Studies were included if they involved crowdsourcing activities, focused on HIV/sexual health, and described the methodology in sufficient detail. We used a content-analytic approach to describe major findings related to types of crowdsourcing models, phases of implementation, advantages, and disadvantages.

Results Our search strategy yielded 431 citations. A total of 17 studies were included for analysis. Four studies were from high-income countries, seven from middle-income countries, one from a low-income country, and five were global. Crowdsourcing was used to develop interventional materials to promote condom use, engage the community in sexual health campaigns, and inform policy changes in sexual health. We identified five types of crowdsourcing models: open contests, hackathons, open forums, extraction of data from social media platforms, and incident reporting systems. Open contests solicit solutions to problems and award prizes to finalists based on predetermined criteria. Hackathons are intensive, approximately 72-hour contests that bring together people to complete a specific task. Crowdsourcing projects shared four common phases: preparation, soliciting crowd inputs, judging submissions, and sharing solutions. Advantages of crowdsourcing included higher potential for innovation due to crowd heterogeneity, encouragement of multisectoral collaboration, empowerment of vulnerable populations, and creation of costeffective and culturally appropriate intervention strategies. Disadvantages included temporal transience and the challenge of sustaining engagement over time.

Conclusion This scoping review revealed diverse applications of crowdsourcing in HIV/sexual health programs and research. Further crowdsourcing research is needed in low and lowermiddle income countries.

Disclosure No significant relationships. 